\title{
The Prognostic Significance of C-Reactive Protein to Albumin Ratio in Newly Diagnosed Acute Myeloid Leukaemia Patients
}

\author{
Liurui Dou (D), Mingyue Shi (D), Juanjuan Song (D), Xiaona Niu (D), Junwei Niu, Shengjie Wei (D), Dan Li, \\ Yanliang Bai, Kai Sun (D) \\ Department of Hematology, Zhengzhou University People's Hospital and Henan Provincial People's Hospital, Henan, People's Republic of China \\ Correspondence: Kai Sun; Yanliang Bai, Department of Hematology, Zhengzhou University People's Hospital and Henan Provincial People's \\ Hospital, No. 7 Weiwu Road, Jinshui District Zhengzhou, Henan, 450003, People's Republic of China, Tel +86-18237II 0038; +86-I37836052II, \\ Email sunkai@cellscience.org; yanliangbai@I26.com
}

Background: The ratio of C-reactive protein to albumin (CAR) is an inflammatory marker that has been demonstrated to be a simple and reliable prognostic factor in several solid tumours and chronic lymphocytic leukaemia (CLL). However, no studies have investigated the prognostic value of the CAR in patients with acute myeloid leukaemia (AML).

Objectives and Methods: We retrospectively analysed 212 newly diagnosed non-M3 AML patients. Using the receiver operating characteristic curve (ROC) method, the optimal cut-off value for CAR was determined. We investigated the correlations of the pretreatment CAR levels with clinical characteristics, treatment response of induction chemotherapy, overall survival (OS) and eventfree survival (EFS). We also assessed the prognostic value of the CAR compared with other inflammation-based prognostic parameters by the area under the curve (AUC).

Results: According to the ROC curve, the optimal cut-off value of CAR was 1.015. CAR was associated with age, C-reactive protein (CRP) levels, albumin levels, ferritin levels, bone marrow blast percentage, French-American-British (FAB) classification, immunophenotype and 2017 European Leukemia Net (2017 ELN) risk stratification. Importantly, we found that high CAR was a powerful indicator of a lower complete remission (CR) rate $(p<0.001)$, worse OS $(p<0.001)$ and worse EFS $(p<0.001)$. Subgroup analysis showed that a high CAR was associated with shorter OS and EFS in patients with intermediate risk stratification or those aged $\leq 65$ years or those without haematopoietic stem cell transplantation (HSCT). In the multivariate analysis, the CAR was an independent prognostic factor for OS and EFS. Furthermore, the predictive value of CAR for OS is superior to that of CRP, albumin and GPS in de novo AML patients aged $\leq 65$ years old.

Conclusion: CAR is a simple and effective prognostic marker in patients with AML. It could be an additional prognostic factor that help further precise the current risk stratification of non-M3 AML, particularly for patients in intermediate risk stratification and those aged $\leq 65$ years and those who did not undergo HSCT.

Keywords: acute myeloid leukaemia, C-reactive protein to albumin ratio, prognosis

\section{Introduction}

Acute myeloid leukaemia (AML) is an aggressive haematological malignancy with a high degree of heterogeneity. ${ }^{1}$ Although advances in treatment have been made in recent years, the long-term survival remains relatively unsatisfactory, with more than $50 \%$ of patients eventually dying from the disease. ${ }^{2}$ Currently, the choice of the best treatment option for AML patients relies on the prognostic risk stratification of the most widely used 2017 European Leukemia Net (2017 ELN), which is mainly based on cytogenetic risk factors and molecular markers. ${ }^{3}$ Although the relationship between some molecular abnormalities and the prognosis of AML has been identified, there remain many molecular abnormalities with relationships with the prognosis of AML that are unclear. This classification does not completely reflect the heterogeneity within each subgroup, especially for AML in the intermediate risk stratification. Therefore, a vital step 
to improve risk stratification is to identify additional prognostic markers that can contribute to clinical decision-making and help to individualize treatments for AML patients.

It has long been recognized that systemic inflammation is associated with tumorigenesis, progression and metastasis. ${ }^{4}$ Although some AML patients have severe infections at the time of presentation, few data are available to assess whether prior infections play a role in the pathogenesis and prognosis of AML. C-reactive protein (CRP) is an acute phase protein produced in the liver following stimulation by cytokines, especially IL-6, and it plays a regulatory role in the inflammatory response by enhancing the function of phagocytes and activating complement. ${ }^{5}$ Investigators have revealed that elevated CRP levels were associated with worse survival outcomes in patients with various types of cancer, including haematologic malignancies. ${ }^{6-8}$ Serum albumin is a measure of an individual's nutritional status. Systemic inflammation can reduce serum albumin concentrations by increasing capillary permeability. ${ }^{9}$ Notably, more than half of cancer patients will develop malnutrition at some point during their illness. The levels of inflammatory factors in malnourished patients are significantly elevated, which could place the body in a state of chronic immunosuppression and ultimately lead to a poor prognosis in cancer patients. ${ }^{10}$ Recently, several studies have shown that hypoalbuminemia is an important independent risk factor for treatment-related morbidity and mortality and inferior survival in AML. ${ }^{11-13}$

The ratio of CRP to albumin (CAR) is a useful score based on inflammation and nutrition, reflecting not only systemic inflammation status but also nutritional status. CAR has recently been proved to be a prognostic marker for various solid tumours ${ }^{14-16}$ and haematologic malignancies, such as chronic lymphocytic leukaemia. ${ }^{17}$ Similarly, the Glasgow prognostic score (GPS) and modified GPS (mGPS), determined by the serum CRP and albumin levels, have been proposed as prognostic indicators for AML patients. ${ }^{18}$ However, the clinical correlations of CAR and its prognostic value in AML patients have not yet been clarified. Therefore, we retrospectively investigated whether CAR has prognostic potential in patients with newly diagnosed non-M3 AML. We also compared the prognostic value of CAR with other established prognostic parameters based on inflammation.

\section{Patients and Methods}

\section{Patients}

This retrospective, single-centre study enrolled 212 newly diagnosed non-M3 AML patients. De novo and secondary AML were included. The samples were collected from January 2017 to December 2020 at Zhengzhou University People's Hospital. The last follow-up occurred in July 2021. Patients who had received prior chemotherapy, had an Eastern Cooperative Oncology Group (ECOG) performance score of $>3$ and were intolerant of chemotherapy, or did not receive induction chemotherapy for other reasons were excluded. All baseline characteristics were collected from the medical records, including age, sex, frequent comorbidities (including hypertension, diabetes mellitus and cardiaccerebral vascular disease), haemoglobin (HB), white blood cell count (WBC), platelet (PLT) count, lactate dehydrogenase (LDH) (normal ranges: 120-250 U/L), ferritin (normal ranges: 11-306 ng/mL), CRP (normal ranges: 0-10 mg/L), albumin (normal ranges: 40-55 g/L), peripheral blood blast percentage, bone marrow blast percentage, immunophenotype and French-American-British (FAB) subtypes. It is worth noting that laboratory data were collected within 24 hours after the first admission.

The CAR was calculated using CRP and albumin concentrations. GPS and mGPS were calculated as previously described. ${ }^{19}$ The GPS values were defined as CRP $\leq 10 \mathrm{mg} / \mathrm{L}$ and albumin $\geq 35 \mathrm{~g} / \mathrm{L}$, scoring 0 point; $\mathrm{CRP}>10 \mathrm{mg} / \mathrm{L}$ or albumin $<35 \mathrm{~g} / \mathrm{L}$, scoring 1 point; and CRP $>10 \mathrm{mg} / \mathrm{L}$ and albumin $<35 \mathrm{~g} / \mathrm{L}$, scoring 2 points. The mGPS was derived from GPS, and the main difference between mGPS and GPS was that only patients with albumin $<35 \mathrm{~g} / \mathrm{L}$ had a score of 0 . This study was conducted in accordance with the Declaration of Helsinki and was approved by the Medical Ethical Committee of Zhengzhou University People's Hospital. Each participant signed a written informed consent before enrolment, and patients younger than 18 years of age were provided with informed consent by their parents or legal guardian.

\section{Treatment and Follow-Up}

Induction chemotherapy regimens included IA (idarubicin $8-12 \mathrm{mg} / \mathrm{m}^{2}$ for 3 days combined with cytarabine $100-200 \mathrm{mg} / \mathrm{m}^{2}$ for 7 days), DA (daunorubicin $40-60 \mathrm{mg} / \mathrm{m}^{2}$ for 3 days combined with cytarabine $100-200 \mathrm{mg} / \mathrm{m}^{2}$ for 7 days), MA 
(mitoxantrone 6-8 mg/m ${ }^{2}$ for 3 days combined with cytarabine $100-200 \mathrm{mg} / \mathrm{m}^{2}$ for 7 days) or CAG $\pm \mathrm{D}$ (aclarubicin $\mathrm{mg} / \mathrm{m}^{2} / \mathrm{d}$ for 4 days, cytarabine $10 \mathrm{mg} / \mathrm{m}^{2} / \mathrm{q} 12 \mathrm{~h}$ for 14 days, G-CSF $300 \mu \mathrm{g} / \mathrm{d}$ for 14 days with/without decitabine $20 \mathrm{mg} / \mathrm{m}^{2} / \mathrm{d}$ for 5 days). Patients with complete remission (CR) continued consolidation chemotherapy with medium-dose or high-dose cytarabine. Haematopoietic stem cell transplantation (HSCT) was performed in patients with moderate or poor prognosis with suitable donors.

\section{Definitions of Treatment Response and Survival Time}

The response criteria were defined as recommended by the international panel of experts on behalf of the 2017 ELN. ${ }^{3}$ Complete remission (CR) was defined as less than 5\% bone marrow blasts, no extramedullary disease, absolute neutrophil count $>1.0 \times 10^{9} / \mathrm{L}$, platelet count $>100 \times 10^{9} / \mathrm{L}$, and independence from red cell transfusions. CR with incomplete haematologic recovery (CRi) was defined as meeting all of the CR criteria except for residual neutropenia $<1.0 \times 10^{9} / \mathrm{L}$ or thrombocytopenia $<100 \times 10^{9} / \mathrm{L}$. Treatment-related mortality (TRM) was defined as death during disease treatment period due to various complications, such as infection, bleeding and organ dysfunction. The endpoints of this study included overall survival (OS), which was calculated from the date of diagnosis to the date of death or the last follow-up, and event-free survival (EFS), which was calculated from the date of diagnosis to the date of primary refractory disease, or relapse from $\mathrm{CR}$, or $\mathrm{CRi}$, or death or the last follow-up.

\section{Statistical Analysis}

All statistical analyses were completed using SPSS software, version 22.0. Categorical variables were compared using the chi-square test or Fisher's exact test. Continuous variables following a normal distribution are expressed as the mean \pm standard deviation (SD), and nonnormally distributed variables are expressed as the median and quartile. The independent-samples $t$-test or Wilcoxon's rank sum test was used for comparisons between consecutive data groups. The cut-off value of CAR was determined using the receiver operating characteristic curve (ROC) method, and the area under the curve (AUC) was calculated to evaluate the discriminatory ability of each prognostic parameter. The KaplanMeier method was used to analyse OS and EFS, and the Log rank test was used to compare the differences between groups. Univariate and multivariate Cox proportional hazards regression was used to analyse factors affecting OS and EFS. The statistical significance level was 0.05 for a two-tailed test.

\section{Results}

\section{Baseline Characteristics}

We enrolled 212 patients with newly diagnosed non-M3 AML receiving at least two cycles of induction chemotherapy, of which cases $91.5 \%$ were identified as de novo and $8.5 \%$ as secondary. The baseline characteristics are summarized in Table 1. Among the 212 patients, 120 (56.6\%) were men. The median age at diagnosis was 49 years old (range 7-82 years). All patients were tested for cytogenetic and molecular abnormalities of bone marrow blasts, including NPMI, CEBPA, FLT3-ITD, KMT2A, GATA2, ASXL1, RUNX1 and TP53 at diagnosis. According to the risk stratification based on the $2017 \mathrm{ELN}, 20.3 \%, 45.8 \%$, and $33.9 \%$ of the patients had a risk status of favourable, intermediate, and adverse, respectively. The median follow-up time from diagnosis was 25 months (range 1-52 months). During this period, a total of 68 patients (31.6\%) experienced relapse, and 90 patients (42.4\%) died. A total of 212 eligible patients were divided into the high CAR group and the low CAR group based on the optimal cut-off value of CAR.

\section{The Optimal Cut-off Values of CAR and Associations with Clinicopathologic Variables}

According to ROC curve analysis, the optimal cut-off value for CAR to predict treatment response after two cycles of induction chemotherapy (CR1 or non-CR1) was 1.015, and the AUC was 0.690 (95\% CI: $0.525-0.699, p<0.001)$. A total of 114 patients (53.8\%) with CAR $\leq 1.015$ at diagnosis were defined as the low CAR group, and 98 patients (46.2\%) with CAR $>1.015$ at diagnosis were defined as the high CAR group. There were no significant differences in sex, frequent comorbidities, WBC, HB levels, PLT count, LDH levels, peripheral blood blast percentage or TRM between the two groups. Patients in the low CAR group were younger $(p<0.001)$, had lower CRP levels $(p<0.001)$ and had higher albumin 
Table I Patient Demographics and Baseline Characteristics

\begin{tabular}{|c|c|c|c|c|}
\hline Characteristics & All Patients & Low CAR & High CAR & $P$ value \\
\hline Number of patients, $n$ & 212 & 114 & 98 & - \\
\hline Age (years) & $49(7-82)$ & $46(7-82)$ & $54(10-82)$ & $<0.001$ \\
\hline Gender, n (\%) & & & & 0.327 \\
\hline Male & $120(56.6 \%)$ & 61 (53.5\%) & 59 (60.2\%) & \\
\hline Female & $92(43.4 \%)$ & $53(46.5 \%)$ & $39(39.8 \%)$ & \\
\hline Type of AML, n (\%) & & & & 0.021 \\
\hline De novo AML & 194 (91.5\%) & $109(95.6 \%)$ & 85 (86.7\%) & \\
\hline Secondary AML & $18(8.5 \%)$ & $5(4.4 \%)$ & $13(13.3 \%)$ & \\
\hline $\mathrm{HB}(\mathrm{g} / \mathrm{L})$ & $77.9 \pm 21.2$ & $79.1 \pm 21.4$ & $76.4 \pm 20.9$ & 0.358 \\
\hline WBC $\left(\times 10^{9} / \mathrm{L}\right)$ & $\mid 2.03(3.4-4 \mid .7)$ & $10.9(4.0-34.0)$ & $13.9(2.6-55.2)$ & 0.764 \\
\hline PLT Count $\left(\times 10^{9} / \mathrm{L}\right)$ & $36.0(19.3-72.8)$ & $34.0(18-74.5)$ & $37.0(20.0-70.5)$ & 0.765 \\
\hline LDH (U/L) & $389(99-6318)$ & 387 (133-2989) & $417(99-6318)$ & 0.837 \\
\hline CRP (mg/L) & $30.5(9.4-78.7)$ & $10.4(2.8-21.5)$ & $84.1(52.4-134.1)$ & $<0.001$ \\
\hline Albumin $(g / L)$ & $35.9 \pm 5.5$ & $38.6 \pm 4.7$ & $32.8 \pm 4.6$ & $<0.001$ \\
\hline Ferritin $(\mathrm{ng} / \mathrm{mL})$ & $439.7(250.6-761.7)$ & $338.6(122.1-632.5)$ & $518.9(414.9-1154.9)$ & 0.001 \\
\hline Bone Marrow Blasts (\%) & $59.2(40.4-76.0)$ & $50.8(25.6-71.6)$ & $66.6(42.0-79)$. & 0.026 \\
\hline Peripheral Blood Blasts (\%) & $43.0(13.0-76.0)$ & $35(10.5-73.0)$ & $47.0(17.0-79.6)$ & 0.428 \\
\hline \multicolumn{5}{|l|}{ Comorbidities, n (\%) } \\
\hline Hypertension & 19 (9.0\%) & $7(6.1 \%)$ & $12(12.2 \%)$ & 0.416 \\
\hline Diabetes mellitus & $8(3.8 \%)$ & $4(3.5 \%)$ & $4(4.2 \%)$ & $1.0^{\mathrm{a}}$ \\
\hline Cardiac-cerebral vascular disease & II (I8.9\%) & $3(2.7 \%)$ & $8(8.2 \%)$ & $0.308^{\mathrm{a}}$ \\
\hline Two or more comorbidities & $14(6.7 \%)$ & $9(7.9 \%)$ & $5(5.1 \%)$ & 0.077 \\
\hline \multicolumn{5}{|l|}{ FAB Classification, $n$ (\%) } \\
\hline Mo & $4(1.9 \%)$ & $2(1.7 \%)$ & $2(2.0 \%)$ & $1.0^{\mathrm{a}}$ \\
\hline MI & $8(3.3 \%)$ & $3(2.6 \%)$ & $5(4.1 \%)$ & $0.482^{\mathrm{a}}$ \\
\hline M2 & $117(50.5 \%)$ & 71 (57.9\%) & $46(41.8 \%)$ & 0.025 \\
\hline M4 & $32(15.1 \%)$ & $15(13.2 \%)$ & $17(17.4 \%)$ & 0.444 \\
\hline M5 & $32(14.6 \%)$ & $17(14.9 \%)$ & $15(14.3 \%)$ & 0.936 \\
\hline M6 & I $(0.5 \%)$ & $0(0)$ & I (I.0\%) & $1.0^{\mathrm{a}}$ \\
\hline M7 & $\mathrm{I}(0.5 \%)$ & $\mathrm{I}(0.9 \%)$ & $0(0)$ & $1.0^{\mathrm{a}}$ \\
\hline Unclassifiable & $17(8 \%)$ & $5(4.4 \%)$ & $12(12.2 \%)$ & 0.036 \\
\hline \multicolumn{5}{|l|}{ ELN risk stratification, $n(\%)$} \\
\hline Favorable & $43(20.3 \%)$ & $33(28.9 \%)$ & $10(10.2 \%)$ & 0.001 \\
\hline Intermediate & 97 (45.8\%) & $49(43.0 \%)$ & $48(49.0 \%)$ & 0.409 \\
\hline Adverse & $72(33.9 \%)$ & 32 (28.1\%) & $40(40.8 \%)$ & 0.08 \\
\hline CRI, n (\%) & $160(75.5 \%)$ & $98(86.0 \%)$ & $62(63.3 \%)$ & $<0.001$ \\
\hline Relapse, n (\%) & 68 (42.5\%) & $36(36.7 \%)$ & $32(51.6 \%)$ & 0.064 \\
\hline HSCT, n (\%) & 40 (18.9\%) & 27 (23.7\%) & $13(13.3 \%)$ & 0.053 \\
\hline \multicolumn{5}{|l|}{ TRM, n (\%) } \\
\hline Infection & $28(13.2 \%)$ & $13(11.4 \%)$ & 15 (15.3\%) & 0.102 \\
\hline Bleeding & $12(5.7 \%)$ & $5(4.4 \%)$ & 7 (7.1\%) & 0.685 \\
\hline Organ dysfunction & $5(2.6 \%)$ & $3(2.6 \%)$ & $2(2.0 \%)$ & $0.652^{\mathrm{a}}$ \\
\hline
\end{tabular}

Note: ${ }^{a}$ Fisher's exact test, $p<0.05$ is considered significant.

Abbreviations: CAR, C-reactive protein to albumin ratio; HB, hemoglobin; WBC, white blood cell count; PLT, platelets; LDH, lactate dehydrogenase; CRP, C-reactive protein; FAB, French-American-British; ELN, European Leukemia Net; CRI, first complete remission after two cycles of induction therapy; HSCT, hematopoietic stem cell transplantation; TRM, treatment-related mortality. 
levels $(p<0.001)$. The comparison between the two groups showed obvious differences in ferritin levels and bone marrow blast percentage, which were higher in the high CAR group ( $p=0.001$ and $p=0.026$ ). Additionally, we also observed statistically significant differences in the type of AML, French-American-British (FAB) classification and 2017 ELN risk stratification between the low and high CAR groups, and the proportions of de novo AML ( $p=0.021)$, AML-M2 $(p=0.025)$ and favourable risk stratification $(p=0.001)$ were higher in the low CAR group.

\section{Relationship Between CAR and Immunophenotype}

In our study, the immunophenotyping of bone marrow blast samples at diagnosis was evaluated in 194 patients. The immunophenotypic results are listed in Table 2. In particular, 60 of 108 patients (55.6\%) in the low CAR group were CD15 positive. In contrast, 31 of 86 patients (36\%) were CD15 positive in the high CAR group, and the expression of CD15 was significantly higher in the low CAR group than in the high CAR group $(p=0.007)$. Similarly, we observed that patients in the low CAR group were more likely to have higher expression of CD33 ( $p=0.046)$. However, the expression of other markers, such as CD34, CD38, CD117, HLA-DR, CD13, CD123, CD7, CD11b, CD56, CD64 and CD71, was not significantly different between the two groups (all $p>0.05$ ).

\section{Relationship Between CAR and Treatment Response of Induction Chemotherapy}

For the entire cohort of 212 patients, 160 patients (75.5\%) achieved CR (CR/CRi) after two cycles of induction chemotherapy. Ninety-eight patients $(86.0 \%)$ achieved CR in the low CAR group compared to 62 patients $(63.3 \%)$ in the high CAR group, and the $\mathrm{CR}$ rate in the low CAR group was remarkably higher than that in the high CAR group $(p<0.001)$. Among the 160 patients who experienced CR, 68 patients suffered disease relapse, including 36 in the low CAR group (36.7\%) and 32 in the high CAR group (51.6\%). Patients in the high CAR group presented a trend towards a greater risk of relapse $(p=0.064)$.

\section{Relationship Between CAR and Clinical Outcomes}

The CRP blood concentration that distinguished CR1 from non-CR1 was $19.92 \mathrm{mg} / \mathrm{L}$ (ROC analysis). We verified the association of high CRP and low albumin levels with worse OS and EFS in AML by Kaplan-Meier survival curve analysis (Figure 1A-D). By the end of the follow-up visit, the median follow-up time for the whole cohort was 25

Table 2 Immunophenotypic Analysis of Bone Marrow Blasts Between the Two Groups

\begin{tabular}{|l|l|l|l|l|}
\hline Immunophenotype & Low CAR (n=108) & High CAR (n=86) & $\chi^{2}$ & $P$ value \\
\hline CD34 & $84(77.8 \%)$ & $61(70.9 \%)$ & 1.189 & 0.276 \\
\hline CD38 & $100(92.6 \%)$ & $77(89.5 \%)$ & 0.560 & 0.454 \\
\hline CDII7 & $99(91.7 \%)$ & $73(84.9 \%)$ & 2.191 & 0.139 \\
\hline HLA-DR & $98(90.7 \%)$ & $71(82.6 \%)$ & 2.855 & 0.091 \\
\hline CD33 & $107(99.1 \%)$ & $80(93.0 \%)$ & - & 0.046 \\
\hline CDI3 & $104(96.3 \%)$ & $82(95.3 \%)$ & - & 0.349 \\
\hline CDI23 & $94(87.0 \%)$ & $80(93.0 \%)$ & 1.855 & 0.173 \\
\hline CD7 & $41(37.9 \%)$ & $32(37.2 \%)$ & 0.012 & 0.914 \\
\hline CDIIb & $30(27.8 \%)$ & $20(23.3 \%)$ & 0.628 & 0.428 \\
\hline CDI5 & $60(55.6 \%)$ & $31(36.0 \%)$ & 7.317 & 0.007 \\
\hline CD56 & $40(37.0 \%)$ & $30(34.9 \%)$ & 0.096 & 0.756 \\
\hline CD64 & $69(65.1 \%)$ & $49(60.0 \%)$ & 0.960 & 1.074 \\
\hline CD7I & $11(10.4 \%)$ & $13(15.1 \%)$ & 0.327 \\
\hline
\end{tabular}

Abbreviation: CAR, C-reactive protein to albumin ratio. 
A

Overall survival

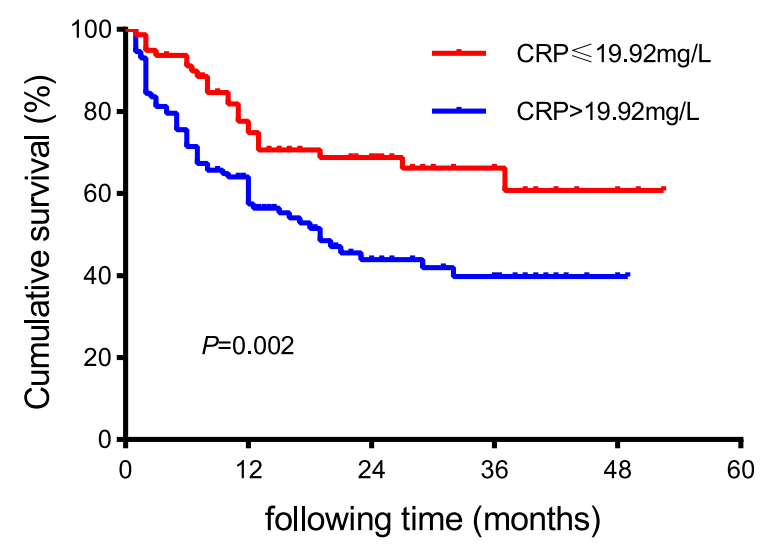

C

Overall survival

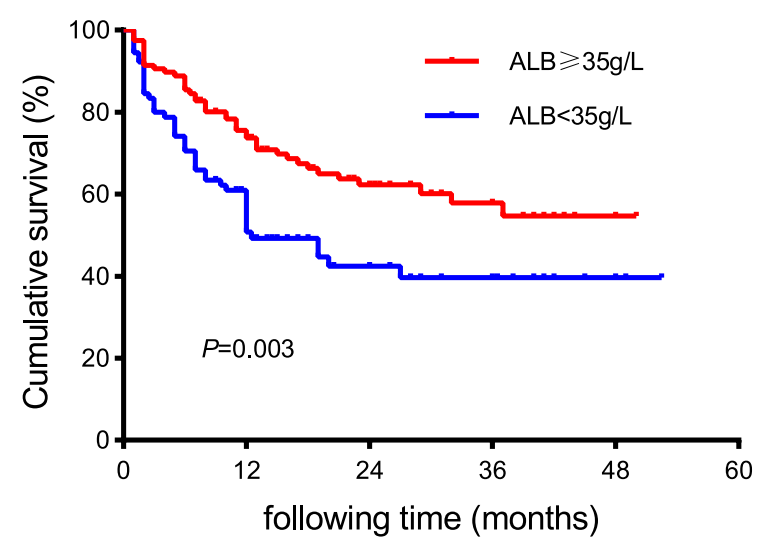

E

Overall survival

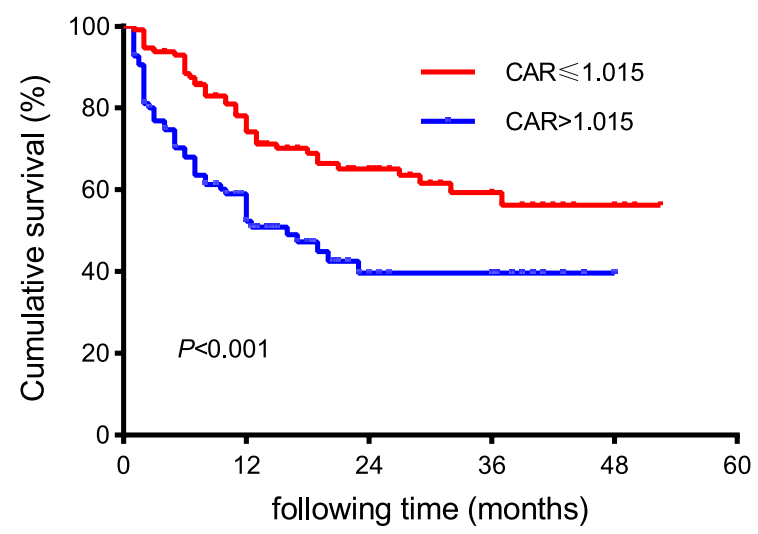

B

Event-free survival

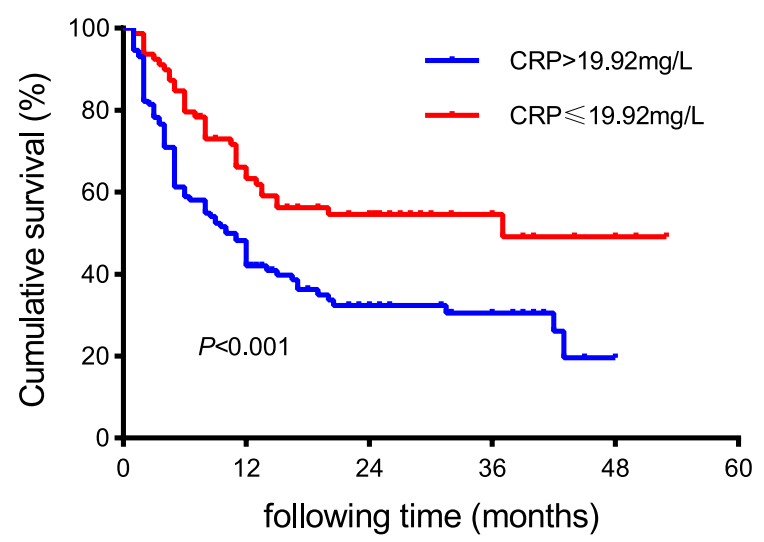

D

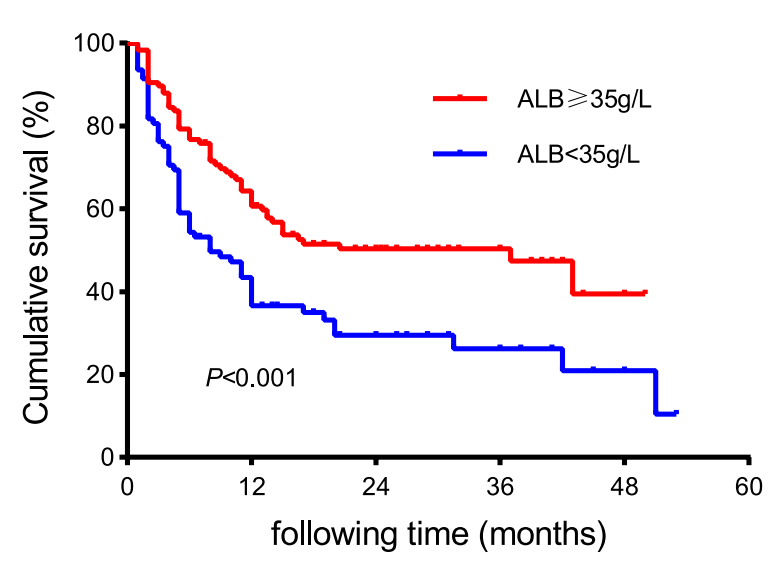

$\mathbf{F}$

Event-free survival

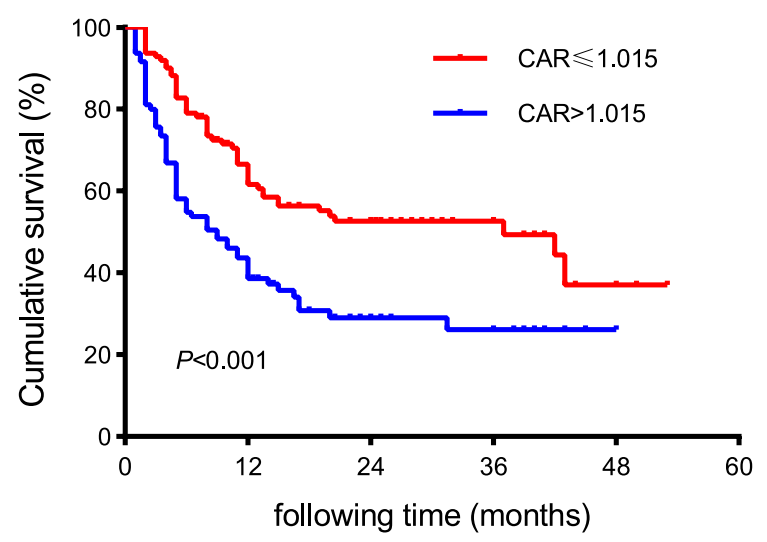

Figure I Kaplan-Meier curves of overall survival (OS) and event-free survival (EFS) for different CRP (A and B), ALB (C and D) and CAR (E and F) levels in AML patients. Abbreviations: CRP, C-reactive protein; ALB, albumin; CAR, C-reactive protein to albumin ratio. 
months (range 1-52 months), the median OS time in the high CAR group was 16 months, and the median OS time in the low CAR group was not reached $(p<0.001)$. Similarly, patients in the high CAR group demonstrated a significantly shorter median EFS (10 vs 37 months; $p<0.001$ ) (Figure 1E and F).

Furthermore, we investigated the relevance of CAR to survival in different subgroups. We investigated whether CAR could further risk stratify patients in each 2017 ELN risk stratification. For patients in the intermediate risk stratification, better median OS ( 37 versus 16 months; $p=0.032$ ) and median EFS ( 37 versus 9 months; $p=0.034$ ) were observed in the low CAR group (Figure 2C and D). However, there were no significant prognostic effects of CAR in patients with favourable and adverse risk stratification (Figure 2A, B, E and F). Additionally, we divided patients into two groups depending on age. Among patients $\leq 65$ years old, we observed that the median OS (not reached vs 20 months; $p=0.003$ ) and median EFS (42 vs 12 months; $p=0.005$ ) were significantly longer in the low CAR group than in the high CAR group (Figure $3 \mathrm{~A}$ and B). In patients $>65$ years old, CAR had no significant effect on prognosis (Figure 3C and D). We also divided patients into two groups based on whether they underwent HSCT. Among patients without HSCT, the median OS (not reached vs 12 months; $p=0.003$ ) and median EFS (20 months vs 8 months; $p<0.001$ ) were significantly better in the low CAR group (Figure 4A and B). However, CAR has no effect on prognosis in patients who underwent HSCT (Figure 4C and D).

\section{Univariate and Multivariable Analyses of Clinical Prognostic Factors}

We analysed various prognostic factors for OS and EFS. The results of the univariate Cox regression analysis are shown in Table 3. In addition to higher CAR (OS: $p=0.001$; EFS: $p=0.002$ ), factors associated with shorter OS and EFS in univariate analysis included age $>65$ (OS: $p<0.001$; EFS: $p<0.001$ ), higher WBC (OS: $p=0.007$; EFS: $p=0.008$ ), higher LDH (OS: $p=0.004$; EFS: $p=0.006$ ) and non-HSCT (OS: $p=0.002$; EFS: $p=0.004)$. Patients with adverse risk stratification showed inferior OS $(p=0.019)$ and EFS $(p=0.009)$. We further investigated the prognostic impact of CAR in the multivariate Cox regression analysis model. In multivariate Cox regression analysis sequentially adjusted for age, WBC count, LDH and adverse risk stratification, a higher CAR remained significantly associated with shorter OS $(p=0.023)$ and EFS $(p=0.034)$ (Table 4).

\section{Comparison of the Prognostic Value of CAR with Other Established Prognostic Parameters in De Novo AML Patients Aged $\leq 65$ Years}

Because of the significant correlations among CRP, albumin, CAR, GPS and mGPS, we further investigated the prognostic significance of CAR compared to other established prognostic parameters. ROC curves for OS were constructed in de novo AML patients aged $\leq 65$ years, and AUC values were compared to assess the discriminatory ability of each prognostic parameter. The AUC value for OS was 0.612 (95\% CI: $0.525-0.699)$ for the CAR, which was equal to that for mGPS (AUC $=0.612,95 \%$ CI: $0.525-0.700$ ) but was higher than those for CRP (AUC $=0.578,95 \%$ CI: 0.487-0.669), albumin ( $\mathrm{AUC}=0.531,95 \% \mathrm{CI}: 0.438-0.624)$ and GPS (AUC $=0.604,95 \%$ CI: $0.515-0.693)$. This result suggested that CAR is a more powerful predictor than CRP, albumin or GPS in de novo non-M3 AML patients aged $\leq 65$ years (Figure 5).

\section{Discussion}

As a sensitive but nonspecific marker of systemic inflammatory markers, serum C-reactive protein (CRP) is affected by a variety of factors, such as infection, injury, and tumours. In contrast, serum albumin concentration is relatively stable and does not vary with short-term changes in nutrient intake or malnutrition status. Previous studies showed that high serum CRP levels and hypoalbuminemia are associated with poor prognosis in AML patients. ${ }^{11,12,20}$ Therefore, we hypothesized that their combination might be a better prognostic factor than CRP or albumin alone in AML patients.

CAR, as a new and stable prognostic marker, has attracted much attention recently. To our knowledge, this study was the first to explore the CAR in newly diagnosed non-M3 AML patients. As the data showed, a high CAR was associated with unfavourable clinical characteristics and adverse risk stratification, such as older age, higher CRP levels, lower albumin levels and higher bone marrow blast percentage. In addition, we also observed that a high level of ferritin led to 
A

Overall survival

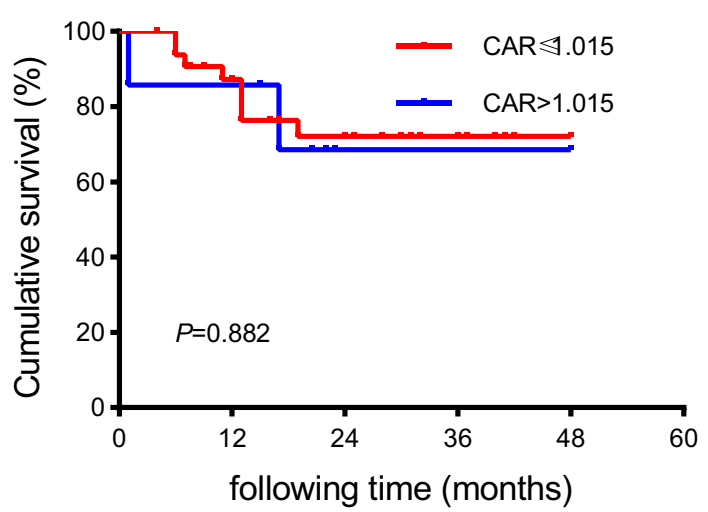

C

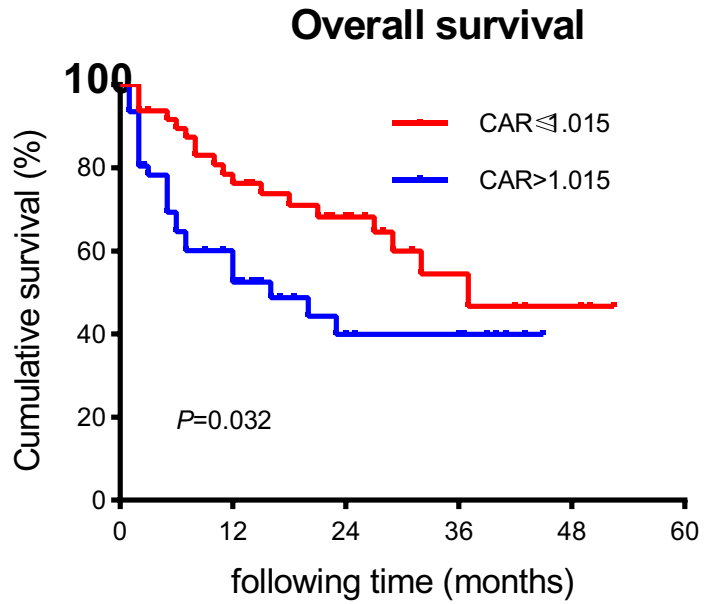

E

Overall survival

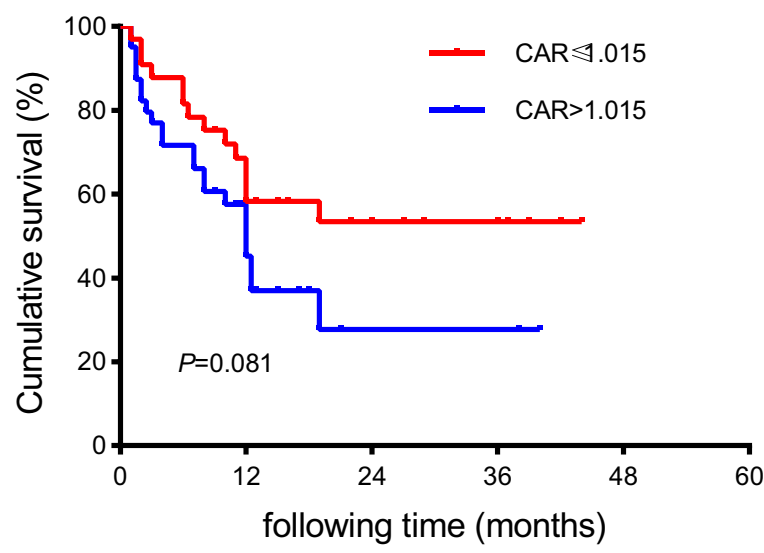

B

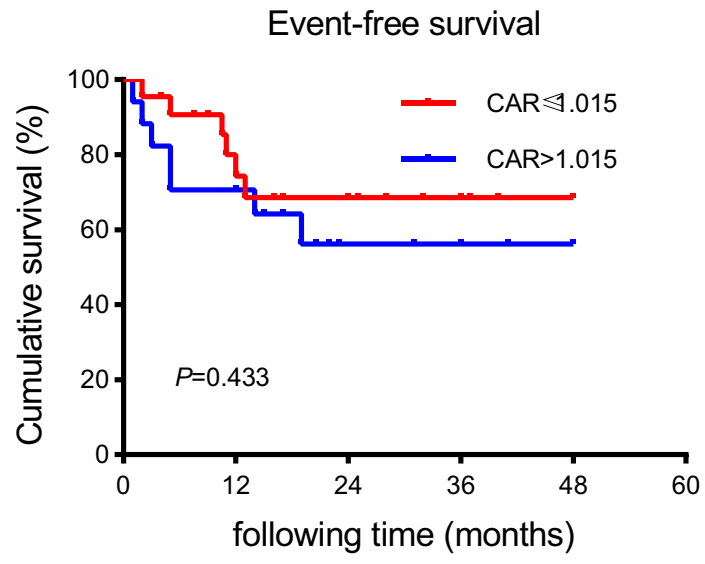

D

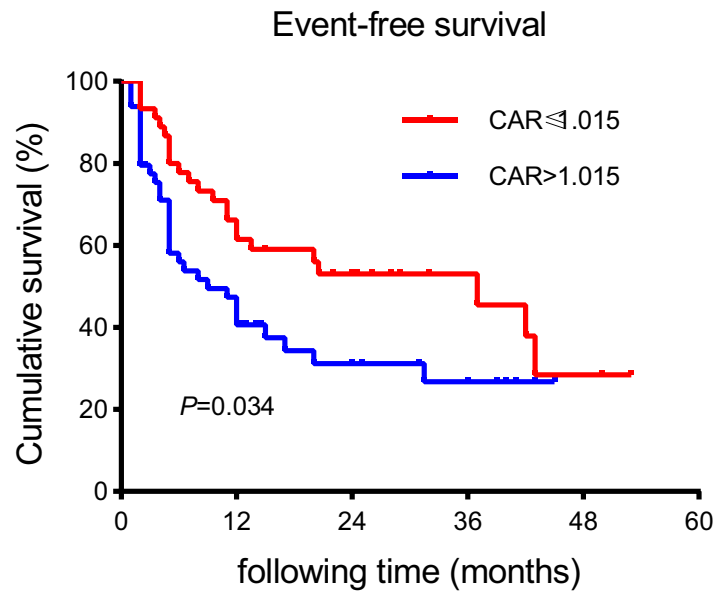

$\mathbf{F}$

Event-free survival

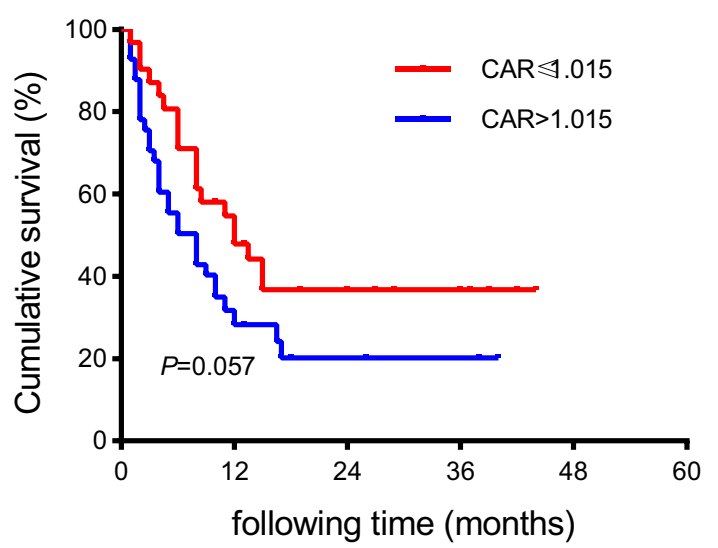

Figure 2 Kaplan-Meier curves of overall survival (OS) and event-free survival (EFS) according to different CAR levels in AML patients with favourable risk (A and B), intermediate risk (C and $\mathbf{D})$, and adverse risk (E and $\mathbf{F})$ stratified by 2017 ELN risk classification.

Abbreviation: CAR, C-reactive protein to albumin ratio. 
A

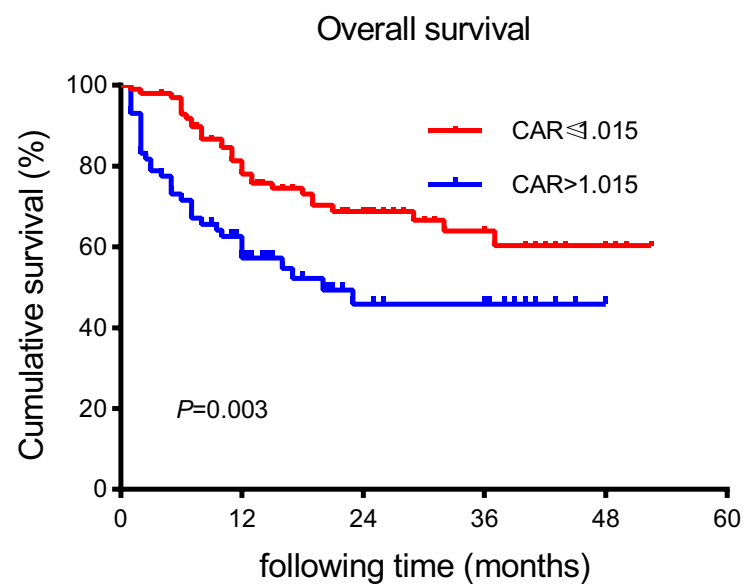

C

Overall survival

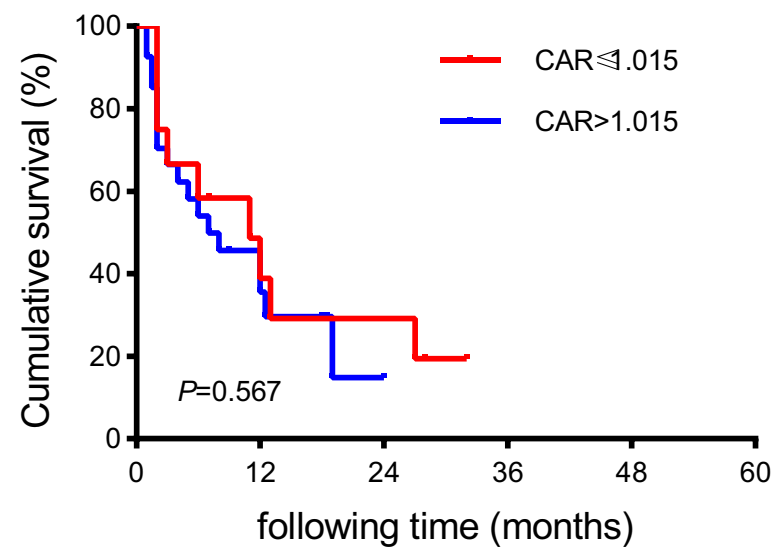

B

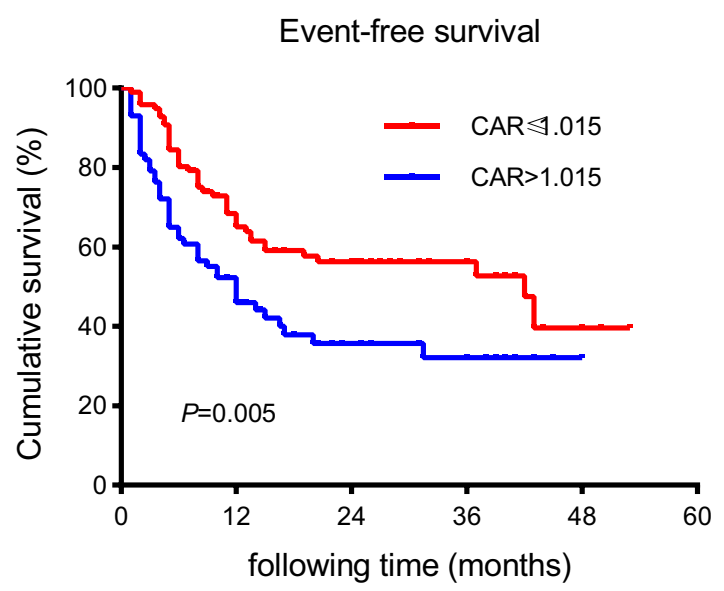

D

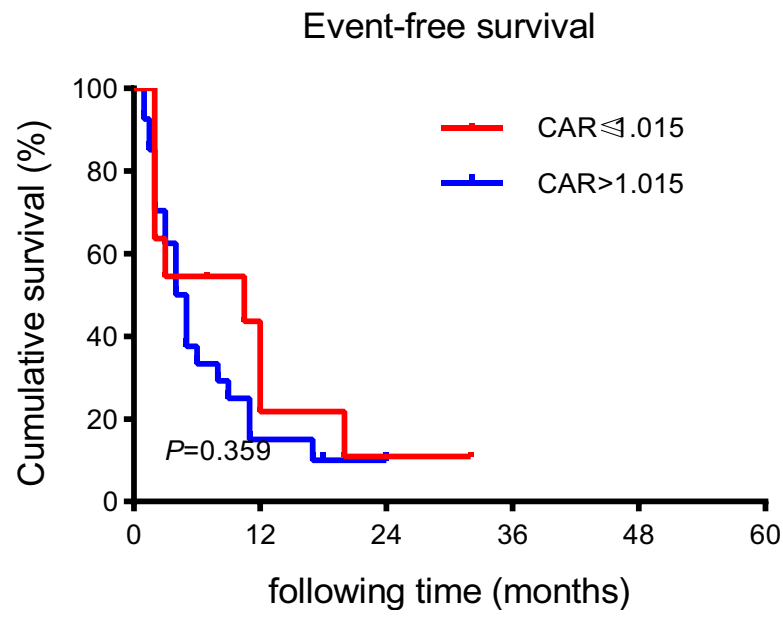

Figure 3 Kaplan-Meier curves of overall survival (OS) and event-free survival (EFS) according to different CAR levels in AML patients $\leq 65$ years old (A and B) and patients $>65$ years old $(\mathbf{C}$ and $\mathbf{D})$ stratified by age.

Abbreviation: CAR, C-reactive protein to albumin ratio.

a high CAR since ferritin also reflects the levels of inflammation. ${ }^{21,22}$ Previous studies have demonstrated that AML patients with high levels of ferritin were associated with higher tumour burden, chemoresistance, relapse and poor prognosis. ${ }^{23-25}$

In our study, a high CAR was significantly associated with worse OS and EFS in the univariate analysis and multivariate analysis, suggesting that CAR was independent of classical prognostic factors, such as age, WBC count, and adverse risk stratification. The present study is consistent with previous studies showing that a high CAR was associated with poor survival. ${ }^{14-16,26}$ Notably, the subgroup analysis that we performed showed that a high CAR was associated with shorter OS and EFS for AML patients in the intermediate risk group. The same result was observed in AML patients aged $\leq 65$ years and who did not undergo HSCT. These results implied that CAR could be an additional prognostic factor that help to further precise the current risk stratification for non-M3 AML, particularly for patients in the intermediate risk group and those aged $\leq 65$ years and those who did not undergo HSCT. To understand which inflammation parameter was more valuable in predicting the survival of de novo non-M3 AML patients $\leq 65$ years old, we directly compared CRP, albumin, GPS and mGPS with CAR by ROC curve analysis. The ROC analysis for OS demonstrated that the AUC value for CAR was higher than those for CRP, albumin and GPS, and it was equal to that for 
A

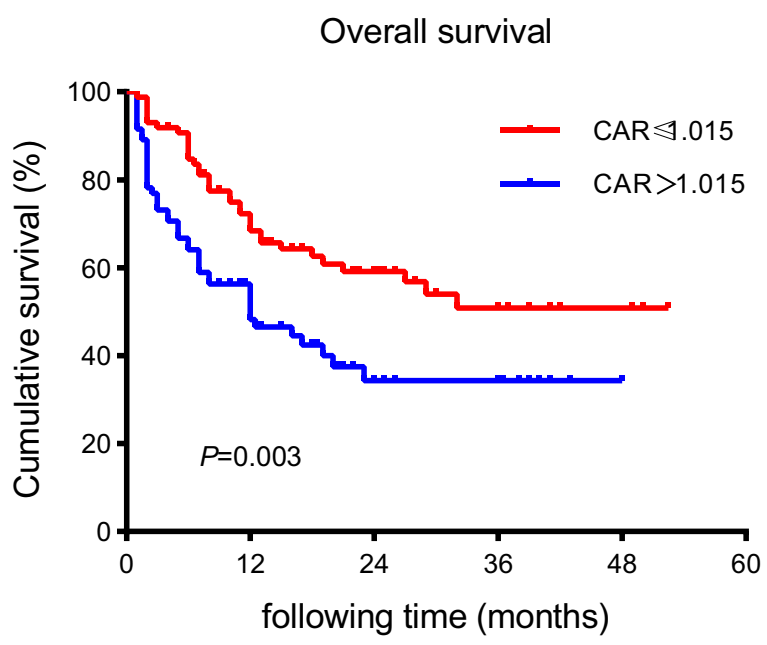

C

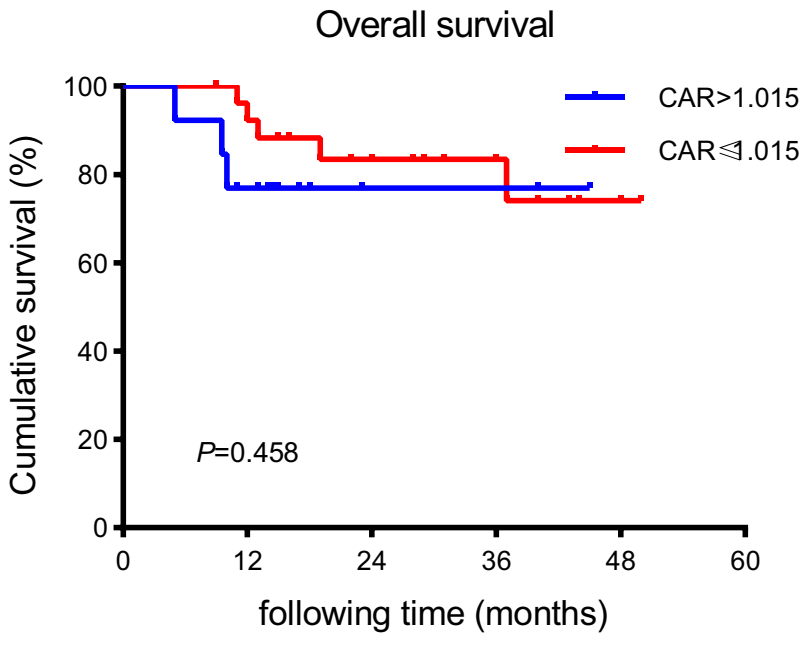

B

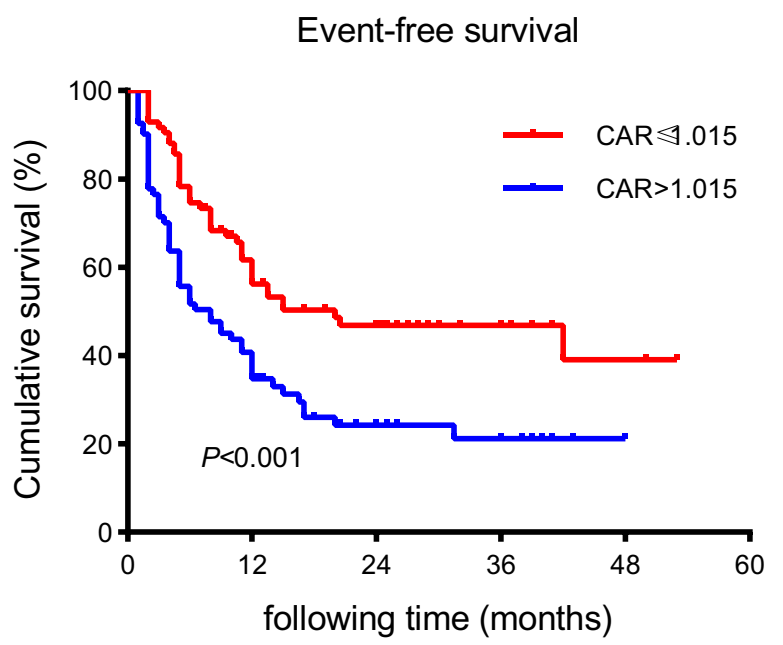

D

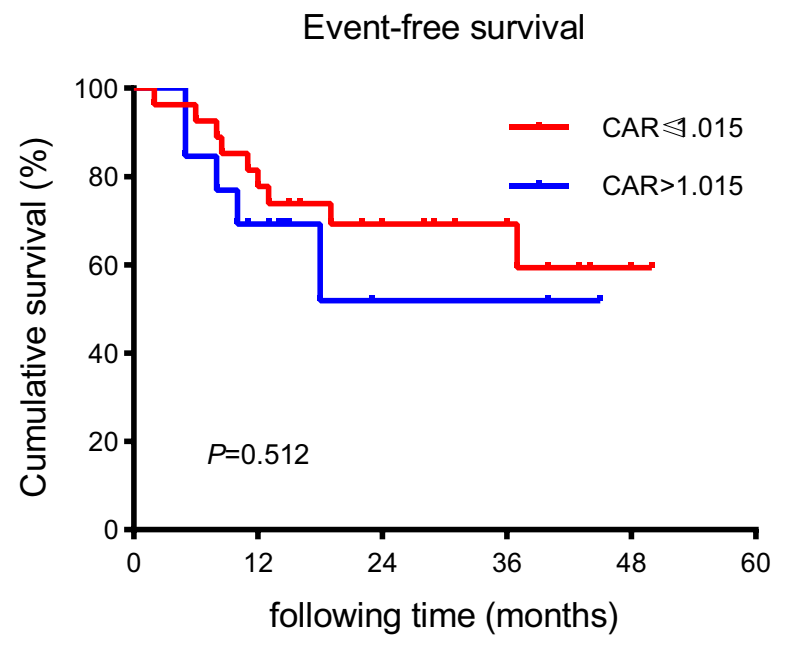

Figure 4 Kaplan-Meier curves of overall survival (OS) and event-free survival (EFS) according to different CAR levels in AML patients without HSCT (A and B) and patients with HSCT (C and D).

Abbreviations: CAR, C-reactive protein to albumin ratio; HSCT, haematopoietic stem cell transplantation.

mGPS, indicating that the predictive value of CAR and mGPS for OS is superior to that of CRP, albumin and GPS in de novo AML patients aged $\leq 65$ years.

In addition, we also observed that patients in the low CAR group had significantly higher CD15 and CD33 expression. The CD15 antigen, a member of the cell adhesion molecule family, is normally expressed on neutrophils. ${ }^{27}$ Previous studies have shown that CD15 expression was associated with a high CR rate ${ }^{28-30}$ and long OS duration. ${ }^{31}$ Repp et al reported that CD33 expression was associated with long OS. ${ }^{32}$ Derolf et al analysed the different expression patterns of CD33 and CD15 in AML patients to predict the clinical outcomes and showed that patients who expressed both CD15 and CD33 had better survival. ${ }^{31}$ We first reported that a low CAR was correlated with the expression of CD15 and CD33, however, the potential association requires further study.

Inflammatory mediators are important components of the tumour local microenvironment and play an important role in the development, chemotherapy resistance and disease progression of AML patients. ${ }^{33,34}$ Inflammatory cytokines, such as IL-6, are able to induce CRP production while suppressing the synthesis of albumin. The levels of IL-6 in peripheral 
Table 3 Univariate Analysis of Overall Survival (OS) and Event-Free Survival (EFS) in AML Patients

\begin{tabular}{|l|l|l|l|l|l|l|}
\hline \multirow{2}{*}{ Variables } & \multicolumn{3}{|c|}{ Overall Survival (OS) } & \multicolumn{3}{c|}{ Event-Free Survival (EFS) } \\
\cline { 2 - 7 } & HR & $\mathbf{9 5 \%} \mathbf{C l}$ & $\mathbf{P}$ value & HR & $\mathbf{9 5 \%}$ Cl & $P$ value \\
\hline Age $>65$ years & 3.092 & $1.952-4.898$ & $<0.001$ & 3.082 & $1.996-4.758$ & $<0.001$ \\
\hline $\mathrm{WBC} \geq 100 \times 10^{9} / \mathrm{L}$ & 2.204 & $1.240-3.918$ & 0.007 & 2.078 & $1.215-3.553$ & 0.008 \\
\hline $\mathrm{HB}<100 \mathrm{~g} / \mathrm{L}$ & 1.701 & $0.851-3.400$ & 0.133 & 1.605 & $0.879-2.932$ & 0.123 \\
\hline $\mathrm{PLT}$ count $<20 \times 10^{9} / \mathrm{L}$ & 1.000 & $0.630-1.588$ & 1.000 & 1.264 & $0.813-1.964$ & 0.298 \\
\hline $\mathrm{LDH} \geq 700 \mathrm{U} / \mathrm{L}$ & 1.905 & $1.232-2.946$ & 0.004 & 1.741 & $1.170-2.591$ & 0.006 \\
\hline ELN risk stratification (adverse vs others) & 1.654 & $1.087-2.516$ & 0.019 & 1.658 & $1.133-2.428$ & 0.009 \\
\hline $\mathrm{CAR}$ & 2.030 & $1.335-3.086$ & 0.001 & 1.816 & $1.247-2.645$ & 0.002 \\
\hline
\end{tabular}

Abbreviations: WBC, white blood cell count; HB, hemoglobin; PLT, platelets; LDH, lactate dehydrogenase; ELN, European Leukemia Net; HSCT, hematopoietic stem cell transplantation; CAR, C-reactive protein to albumin ratio.

Table 4 Multivariate Analysis of Overall Survival (OS) and Event-Free Survival (EFS) in AML Patients

\begin{tabular}{|c|c|c|c|c|c|c|}
\hline \multirow[t]{2}{*}{ Variables } & \multicolumn{3}{|c|}{ Overall Survival (OS) } & \multicolumn{3}{|c|}{ Event-Free Survival (EFS) } \\
\hline & HR & $95 \% \mathrm{Cl}$ & $P$ value & HR & $95 \% \mathrm{Cl}$ & $P$ value \\
\hline Age $>65$ years & 3.553 & $2.089-6.042$ & $<0.001$ & 3.732 & $2.273-6.127$ & $<0.001$ \\
\hline$W B C \geq 100 \times 10^{9} / L$ & 2.312 & I. $186-4.508$ & 0.014 & $2.44 I$ & $1.310-4.55 \mid$ & 0.005 \\
\hline $\mathrm{LDH} \geq 700 \mathrm{U} / \mathrm{L}$ & 2.051 & $1.235-3.406$ & 0.006 & 1.851 & $1.169-2.930$ & 0.009 \\
\hline ELN risk stratification (adverse vs others) & 1.119 & $0.70 \mathrm{I}-1.786$ & 0.638 & 1.162 & $0.760-1.778$ & 0.488 \\
\hline CAR & 1.729 & I.079-2.770 & 0.023 & 1.575 & $1.035-2.398$ & 0.034 \\
\hline
\end{tabular}

Abbreviations: WBC, white blood cell count; LDH, lactate dehydrogenase; ELN, European Leukemia Net; CAR, C-reactive protein to albumin ratio.

blood and bone marrow are significantly elevated in adult AML patients due to autocrine IL- 6 secretion by AML cells or IL-6 production by normal stromal cells or monocytes in the bone marrow. ${ }^{35,36}$ STAT3 signalling is known to regulate antiapoptotic gene expression, and IL-6 can promote chemoresistance and relapse through the STAT3 signalling pathway and support leukaemia stem cells. ${ }^{34}$ Importantly, IL-6 is associated with emotional health of patients, and its levels are closely related to the severity of depression. ${ }^{37}$

However, this study was a single-centre, retrospective study, and the sample size was relatively small in the subgroup analysis. In addition, the heterogeneity of treatment regimens might have confounded the results of this study. AML is a complex and dynamic disease, while our study only involved the association between CAR and the prognosis of AML at the time of diagnosis. Therefore, to further validate the prognostic value of CAR, prospective studies involving large-scale and multi-centre studies should be conducted, and CAR should be monitored dynamically at various time points.

\section{Conclusion}

In conclusion, our results implied that CAR is a simple and effective prognostic marker in patients with AML. It could be an additional prognostic factor that help further precise the current risk stratification of non-M3 AML, particularly for patients in the intermediate risk stratification and those aged $\leq 65$ years and those who did not undergo HSCT. 


\section{Overall survival}

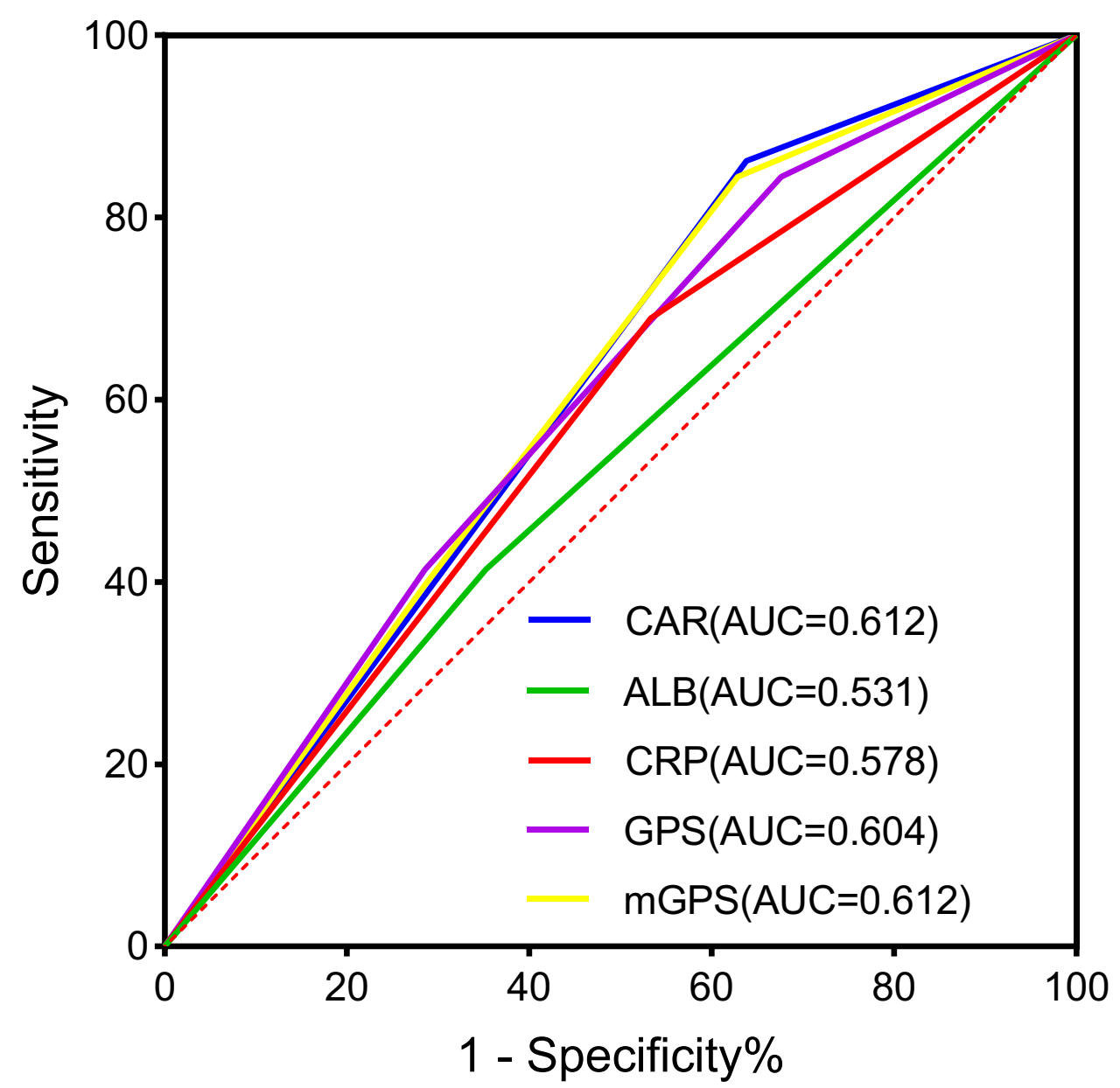

Figure 5 Comparison of the prognostic value of CRP, ALB, GPS, and mGPS with CAR by ROC curves in de novo non-M3 AML patients $\leq 65$ years old. Abbreviations: CRP, C-reactive protein; ALB, albumin; GPS, Glasgow prognostic score; mGPS, modified GPS; CAR, C-reactive protein to albumin ratio.

\section{Ethics Approval and Consent to Participate}

The study protocol was approved by the research ethics committees of Zhengzhou University People's Hospital. Each participant signed a written informed consent form before enrolment, and a parent or legal guardian of patients younger than 18 years of age provided informed consent.

\section{Acknowledgments}

We would like to express our gratitude to all of the doctors, nurses and pathologists of all of the hospitals and haematology departments who participated in this survey. Our manuscript has been edited in English to correct the grammar or style.

\section{Author Contributions}

All of the authors made a significant contribution to the work reported, whether in the conception, study design, execution, acquisition of data, analysis or interpretation or in all of these areas; participated in drafting, revising or critically reviewing the article; gave final approval of the version to be published; agreed on the journal to which the article has been submitted; and agreed to be accountable for all aspects of the work. 


\section{Funding}

This study was partially supported by the National Natural Science Foundation of China (No. 81971508, No. 81471589) and the Foundation and Frontier Research Grant of Henan Provincial Science and Technology Bureau, PR China (No. 112300410027).

\section{Disclosure}

The authors report no conflicts of interest in this work.

\section{References}

1. Juliusson G, Lazarevic V, Horstedt AS, Hagberg O, Hoglund M; Swedish Acute Leukemia Registry G. Acute myeloid leukemia in the real world: why population-based registries are needed. Blood. 2012;119:3890-3899.

2. Short NJ, Rytting ME, Cortes JE. Acute myeloid leukaemia. Lancet. 2018;392:593-606. doi:10.1016/S0140-6736(18)31041-9

3. Dohner H, Estey E, Grimwade D, et al. Diagnosis and management of AML in adults: 2017 ELN recommendations from an international expert panel. Blood. 2017;129:424-447. doi:10.1182/blood-2016-08-733196

4. Diakos CI, Charles KA, McMillan DC, Clarke SJ. Cancer-related inflammation and treatment effectiveness. Lancet Oncol. 2014;15:e493-503. doi:10.1016/S1470-2045(14)70263-3

5. Balkwill F, Mantovani A. Inflammation and cancer: back to Virchow? Lancet. 2001;357:539-545. doi:10.1016/S0140-6736(00)04046-0

6. Barbui T, Carobbio A, Finazzi G, et al. Elevated C-reactive protein is associated with shortened leukemia-free survival in patients with myelofibrosis. Leukemia. 2013;27:2084-2086. doi:10.1038/leu.2013.207

7. Herishanu Y, Polliack A, Shenhar-Tsarfaty S, et al. Increased serum C-reactive protein levels are associated with shorter survival and development of second cancers in chronic lymphocytic leukemia. Ann Med. 2017;49:75-82. doi:10.1080/07853890.2016.1232860

8. Qin W, Yuan Q, Wu J, Yu H, Wang Y, Chen Q. Prognostic value of pre-therapy C-reactive protein level in diffuse large B-cell lymphoma: a meta-analysis. Leuk Lymphoma. 2019;60:358-366. doi:10.1080/10428194.2018.1482540

9. Soeters PB, Wolfe RR, Shenkin A. Hypoalbuminemia: pathogenesis and clinical significance. J Parenter Enteral Nutr. 2019;43:181-193. doi:10.1002/jpen.1451

10. Taniguchi K, Karin M. NF-kappaB, inflammation, immunity and cancer: coming of age. Nat Rev Immunol. 2018;18:309-324. doi:10.1038/ nri.2017.142

11. Wang N, Desai A, Ge B, et al. Prognostic value of hypoalbuminemia at diagnosis in de novo non-M3 acute myeloid leukemia. Leuk Lymphoma. 2020;61:641-649. doi:10.1080/10428194.2019.1686499

12. Lauriane FC, Julien B, Marc M, et al. Serum albumin or body mass index: which prognostic factor for survival in patients with acute myeloblastic leukaemia? Hematol Oncol. 2018;37:80-84

13. Doucette K, Percival ME, Williams L, et al. Hypoalbuminemia as a prognostic biomarker for higher mortality and treatment complications in acute myeloid leukemia. Hematol Oncol. 2021;39(5):697-706.doi:10.1002/hon.2925

14. Ishizuka M, Nagata H, Takagi K, Iwasaki Y, Shibuya N, Kubota K. Clinical significance of the C-reactive protein to albumin ratio for survival after surgery for colorectal cancer. Ann Surg Oncol. 2016;23:900-907. doi:10.1245/s10434-015-4948-7

15. Zhang W, Liu K, Ye B, Liang W, Ren Y. Pretreatment C-reactive protein/albumin ratio is associated with poor survival in patients with stage IB-IIA cervical cancer. Cancer Med. 2018;7:105-113. doi:10.1002/cam4.1270

16. Otowa Y, Nakamura T, Yamamoto M, et al. C-reactive protein to albumin ratio is a prognostic factor for patients with cStage II/III esophageal squamous cell cancer. Dis Esophagus. 2017;30:1-5. doi:10.1093/dote/dox107

17. Tang HN, Pan BH, Wang L, Zhu HY, Li JY. C-reactive protein-to-albumin ratio is an independent poor prognostic factor in newly diagnosed chronic lymphocytic leukaemia: a clinical analysis of 322 cases. Transl Oncol. 2021;14:101035. doi:10.1016/j.tranon.2021.101035

18. Heini AD, Hugo R, Berger MD, Novak U, Bacher U, Pabst T. Simple acute phase protein score to predict long-term survival in patients with acute myeloid leukemia. Hematol Oncol. 2020;38:74-81. doi:10.1002/hon.2696

19. McMillan DC. The systemic inflammation-based Glasgow prognostic score: A decade of experience in patients with cancer. Cancer Treat Rev. 2013;39:534-540. doi:10.1016/j.ctrv.2012.08.003

20. Loh KP, Tooze JA, Nicklas BJ, et al. Inflammatory biomarkers, geriatric assessment, and treatment outcomes in acute myeloid leukemia. J Geriatr Oncol. 2020;11:410-416. doi:10.1016/j.jgo.2019.03.014

21. Manerba M, Di Ianni L, Govoni M, Roberti M, Recanatini M, Di Stefano G. Lactate dehydrogenase inhibitors can reverse inflammation induced changes in colon cancer cells. Eur J Pharm Sci. 2017;96:37-44. doi:10.1016/j.ejps.2016.09.014

22. Kell DB, Pretorius E. Serum ferritin is an important inflammatory disease marker, as it is mainly a leakage product from damaged cells. Metallomics. 2014;6:748-773. doi:10.1039/c3mt00347g

23. Lebon D, Vergez F, Bertoli S, et al. Hyperferritinemia at diagnosis predicts relapse and overall survival in younger AML patients with intermediate-risk cytogenetics. Leuk Res. 2015;39:818-821. doi:10.1016/j.leukres.2015.05.001

24. Tachibana T, Andou T, Tanaka M, et al. Clinical significance of serum ferritin at diagnosis in patients with acute myeloid leukemia: a YACHT multicenter retrospective study. Clin Lymphoma Myeloma Leuk. 2018;18:415-421. doi:10.1016/j.clml.2018.03.009

25. Ihlow J, Gross S, Sick A, et al. AML: high serum ferritin at initial diagnosis has a negative impact on long-term survival. Leuk Lymphoma. 2019;60:69-77. doi:10.1080/10428194.2018.1461860

26. Kinoshita A, Onoda H, Imai N, et al. The C-reactive protein/albumin ratio, a novel inflammation-based prognostic score, predicts outcomes in patients with hepatocellular carcinoma. Ann Surg Oncol. 2015;22:803-810. doi:10.1245/s10434-014-4048-0

27. Skubitz KM, Snook RW 2nd. Monoclonal antibodies that recognize lacto-N-fucopentaose III (CD15) react with the adhesion-promoting glycoprotein family (LFA-1/HMac-1/gp 150,95) and CR1 on human neutrophils. J Immunol. 1987;139:1631-1639. 
28. Campos L, Guyotat D, Archimbaud E, et al. Surface marker expression in adult acute myeloid leukaemia: correlations with initial characteristics, morphology and response to therapy. Br J Haematol. 1989;72:161-166. doi:10.1111/j.1365-2141.1989.tb07677.x

29. Tien HF, Wang CH, Lin MT, et al. Correlation of cytogenetic results with immunophenotype, genotype, clinical features, and ras mutation in acute myeloid leukemia A study of 235 Chinese patients in Taiwan. Cancer Genet Cytogenet. 1995;84:60-68. doi:10.1016/0165-4608(95)00084-4

30. Chisini M, Stefanizzi C, Ceglie T, et al. Independent prognostic impact of CD15 on complete remission achievement in patients with acute myeloid leukemia. Hematol Oncol. 2017;35:804-809. doi:10.1002/hon.2331

31. Derolf AR, Bjorklund E, Mazur J, Bjorkholm M, Porwit A. Expression patterns of CD33 and CD15 predict outcome in patients with acute myeloid leukemia. Leuk Lymphoma. 2008;49:1279-1291. doi:10.1080/10428190802123994

32. Repp R, Schaekel U, Helm G, et al. Immunophenotyping is an independent factor for risk stratification in AML. Cytometry B Clin Cytom. 2003;53:11-19. doi:10.1002/cyto.b.10030

33. Sanchez-Correa B, Bergua JM, Campos C, et al. Cytokine profiles in acute myeloid leukemia patients at diagnosis: survival is inversely correlated with IL-6 and directly correlated with IL-10 levels. Cytokine. 2013;61:885-891. doi:10.1016/j.cyto.2012.12.023

34. Stevens AM, Miller JM, Munoz JO, Gaikwad AS, Redell MS. Interleukin-6 levels predict event-free survival in pediatric AML and suggest a mechanism of chemotherapy resistance. Blood Adv. 2017;1:1387-1397. doi:10.1182/bloodadvances.2017007856

35. Schuringa JJ, Wierenga AT, Kruijer W, Vellenga E. Constitutive Stat3, Tyr705, and Ser727 phosphorylation in acute myeloid leukemia cells caused by the autocrine secretion of interleukin-6. Blood. 2000;95:3765-3770. doi:10.1182/blood.V95.12.3765

36. Reikvam H, Brenner AK, Hagen KM, et al. The cytokine-mediated crosstalk between primary human acute myeloid cells and mesenchymal stem cells alters the local cytokine network and the global gene expression profile of the mesenchymal cells. Stem Cell Res. 2015;15:530-541. doi:10.1016/j.scr.2015.09.008

37. Oliveira Miranda D, Soares de Lima TA, Ribeiro Azevedo L, Feres O, Ribeiro da Rocha JJ, Pereira-da-Silva G. Proinflammatory cytokines correlate with depression and anxiety in colorectal cancer patients. Biomed Res Int. 2014;2014:739650. doi:10.1155/2014/739650

\section{Publish your work in this journal}

Cancer Management and Research is an international, peer-reviewed open access journal focusing on cancer research and the optimal use of preventative and integrated treatment interventions to achieve improved outcomes, enhanced survival and quality of life for the cancer patient. The manuscript management system is completely online and includes a very quick and fair peer-review system, which is all easy to use. Visit http://www.dovepress.com/testimonials.php to read real quotes from published authors.

Submit your manuscript here: https://www.dovepress.com/cancer-management-and-research-journa 